\title{
Attitudes toward assisted suicide requests in the context of severe and persistent mental illness: A survey of psychiatrists in Switzerland
}

\author{
Hodel, Martina A ; Hoff, Paul ; Irwin, Scott A ; Biller-Andorno, Nikola ; Riese, Florian ; Trachsel, \\ Manuel
}

\begin{abstract}
Objective: Switzerland is among the few countries worldwide where a request for assisted suicide (AS) can be granted on the basis of a primary psychiatric diagnosis. Psychiatrists play an increasingly important role in this regard, especially when the request for AS arises in the context of suffering caused by severe and persistent mental illness (SPMI). The objective of the survey was to assess general attitudes among psychiatrists in Switzerland regarding AS requests from patients with SPMI. Method: In a cross-sectional survey of 1,311 German-speaking psychiatrists in Switzerland, participants were asked about their attitude to AS for patients with SPMI, based on three case vignettes of patients diagnosed with anorexia nervosa, treatment-refractory depression, or severe persistent schizophrenia. Result: From a final sample of 457 psychiatrists (a response rate of 34.9\%) whose mean age was 57.8 years, $48.6 \%$ of respondents did not support access to AS for persons diagnosed with SPMI, $21.2 \%$ were neutral, and $29.3 \%$ indicated some degree of support for access. In relation to the case vignettes, a slightly higher percentage of respondents supported the patient's wish to seek AS: $35.4 \%$ for those diagnosed with anorexia nervosa, $32.1 \%$ for those diagnosed with depression, and $31.4 \%$ for those diagnosed with schizophrenia. Significance of results: Although a majority of the responding psychiatrists did not support AS for SPMI patients, about one-third would have supported the wishes of patients in the case vignettes. In light of the increasing number of psychiatric patients seeking AS and the continuing liberalization of AS practices, it is important to understand and take account of psychiatrists' perspectives. Keywords: Assisted suicide; medical aid in dying; rational suicide; severe and persistent mental illness; treatment resistance.
\end{abstract}

DOI: https://doi.org/10.1017/s1478951519000233

Posted at the Zurich Open Repository and Archive, University of Zurich ZORA URL: https://doi.org/10.5167/uzh-211897

Journal Article

Accepted Version

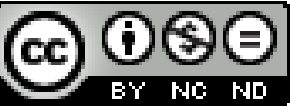

The following work is licensed under a Creative Commons: Attribution-NonCommercial-NoDerivatives 4.0 International (CC BY-NC-ND 4.0) License.

Originally published at:

Hodel, Martina A; Hoff, Paul; Irwin, Scott A; Biller-Andorno, Nikola; Riese, Florian; Trachsel, Manuel (2019). Attitudes toward assisted suicide requests in the context of severe and persistent mental illness: 
A survey of psychiatrists in Switzerland. Palliative Supportive Care, 17(6):621-627. DOI: https://doi.org/10.1017/s1478951519000233 
Title: Attitudes towards assisted suicide requests in the context of severe and persistent mental illness: A survey among psychiatrists in Switzerland

Short title/running head: Attitudes towards assisted suicide requests

\section{Authors:}

1 Martina A. Hodel (first and corresponding)

2 Paul Hoff

3 Scott A. Irwin

4 Nikola Biller-Andorno

5 Florian Riese

6 Manuel Trachsel (last)

\section{Authors' affiliations}

$1^{\text {st }}$ Martina A. Hodel, MSc, Institute of Biomedical Ethics and History of Medicine, University of Zurich, Winterthurerstrasse 30, 8006, Zurich, Switzerland, martina.hodel@ibme.uzh.ch,+41446348368

$2^{\text {nd }}$ Paul Hoff, MD, PhD, Psychiatric University Hospital Zurich, Lenggstrasse 31, 8008, Zurich, Switzerland

$3^{\text {rd }}$ Scott A. Irwin, MD, PhD, Cedars-Sinai Health System, 8700 Beverly Blvd, Los Angeles, CA 90048

$4^{\text {th }}$ Nikola Biller-Andorno, MD, PhD, Institute of Biomedical Ethics and History of Medicine, University of Zurich, Winterthurerstrasse 30, 8006, Zurich, Switzerland

$5^{\text {th }}$ Florian Riese, MD, Psychiatric University Hospital Zurich, Division of Psychiatry Research and Psychogeriatric Medicine, Lenggstrasse 31, 8008, Zurich, Switzerland $6^{\text {th }}$ Manuel Trachsel, MD, PhD, Institute of Biomedical Ethics and History of Medicine, University of Zurich, Winterthurerstrasse 30, 8006, Zurich, Switzerland

\section{Details}

Total pages of this manuscript: 17 (including references, without tables and figures)

Number of tables: 3

Number of figures: 3 


\title{
Title: Attitudes towards assisted suicide requests in the context of severe and persistent mental illness: A survey among psychiatrists in Switzerland
}

\begin{abstract}
(word count: 250)
Objective Switzerland is among the few countries worldwide where requests for assisted suicide (AS) can be granted based on a primary psychiatric diagnosis. Psychiatrists play an increasingly important role for requests of AS, especially when the requests are based on the suffering from severe and persistent mental illness (SPMI). The objective of this survey among psychiatrists in Switzerland was to assess general attitudes regarding AS requests from patients with SPMI and regarding three different case vignettes of patients diagnosed with SPMIs.
\end{abstract}

Methods A cross-sectional survey among 1311 German-speaking psychiatrists in Switzerland was performed. Participants were asked about their general support regarding AS for patients with SPMI and about three specific case vignettes of patients diagnosed with either anorexia nervosa, treatment-refractory depression, or severe persistent schizophrenia.

$\underline{\text { Results }}$ The response rate was $34.9 \%(n=457)$. Mean age of the respondents was 57.8 years. $48.6 \%$ of the respondents did not support access to AS for persons diagnosed with SPMI, while $21.2 \%$ of respondents remained neutral, and $29.3 \%$ stated that they do support access to some degree. A slightly higher percentage of the participating psychiatrists would have supported the patients in the case vignettes in their wish to seek AS (35.4\% for the patients diagnosed with anorexia nervosa; $32.1 \%$ for the patient diagnosed with depression, and $31.4 \%$ for the patient diagnosed with schizophrenia).

Significance of the Results A majority of the responding psychiatrists did not support AS for SPMI patients. Around a third, however, would have supported the patients in the specific case vignettes in their wish to seek AS. Considering the increasing number of psychiatric patients seeking AS and the continuing liberalization of AS practices, it is important to understand and include psychiatrists' perspectives.

\section{Keywords}

- $\quad$ assisted suicide

- medical aid in dying

- $\quad$ severe and persistent mental illness

- treatment resistance

- rational suicide 


\section{Introduction}

Assisted suicide ${ }^{1}$ (AS) is now legal in some countries in Europe, certain U.S. states, Canada, Colombia, and the Australian state of Victoria starting from 2019 (Dyer, White, \& Rada, 2015; Emanuel, Onwuteaka-Philipsen, Urwin, \& Cohen, 2016). Traditionally, AS has evolved as a medical intervention, performed by physicians and reserved for the terminally ill population in order to prevent unnecessary suffering and respect for patient autonomy. There is wide support in Western societies for AS by both the public and medical professionals (Emanuel et al., 2016); however, the degree of support is greatly dependent on the specific patient group. Specifically, surveys have shown that physicians, including psychiatrists, are generally more reluctant towards AS if the request is based on the suffering from mental illness compared to suffering from physical illness (Rousseau, Turner, Chochinov, Enns, \& Sareen, 2017; Bolt, Snijdewind, Willems, Heide, \& Onwuteaka-Philipsen, 2015; Brauer, Bolliger, \& Strub, 2015; Kouwenhoven et al., 2013). AS on the basis of a mental illness is legal only in a few jurisdictions and remains particularly controversial (Miller \& Appelbaum, 2018; Vandenberghe, 2018; Appelbaum, 2016; S. Kim \& Lemmens, 2016; Schuklenk \& Vathorst, 2015). There, however, the number of persons receiving AS for psychiatric disorders is increasing. In the Netherlands, 13 cases were reported in 2011, while the number increased to 60 in 2016 and 83 in 2017 (Regional euthanasia review committees, 2017). Due to a missing reporting system in Switzerland, there is a lack of information on Swiss psychiatric AS cases. However, there is a strong increase in AS since 2008, resulting in an increase of absolute number of non-terminal patients seeking AS (Steck, Junker, \& Zwahlen, 2018). The Swiss model differs significantly from other approaches in a few ways (see summary in box 1). Worldwide, Switzerland is the only country where the legal framework

\footnotetext{
${ }^{1}$ In the course of the legalization and depending on the respective predominant concepts, several different terms have been established, assisted suicide and (medically) assisted dying among others. While being aware of the controversy surrounding the different terms, the authors use the term assisted suicide because it is typically utilized in Switzerland.
} 
neither requires a terminal (somatic) condition for patients requesting AS nor foresees a specific role for physicians in the process of the request. Specifically, the physician's involvement is limited to the assessment of decision-making capacity (DMC) and the prescription of the lethal drug, and there are no further legal regulations regarding access and procedure (Gamondi, Borasio, Oliver, Preston, \& Sheila Payne, 2017; Ziegler \& Bosshard, 2007, p.). In practice, non-governmental 'right-to-die' organizations (RTDOs) evaluate, coordinate and respond to AS requests (Ziegler, 2009; Ziegler \& Bosshard, 2007). The RTDOs define internal guidelines specifying the necessary preconditions and procedural requirements, including whether requests can be made on the basis of suffering from a mental illness (Ziegler \& Bosshard, 2007). Criticism notwithstanding, the Swiss model has been described as de-medicalizing AS and resolving common ethical dilemmas experienced by medical professionals (Ziegler, 2009). Up to now, AS in Switzerland has ostensibly been treated as a political and public concern rather than a medical one. Nevertheless, many hospitals, nursing homes, hospices, and other institution involved in the care of seriously ill patients in Switzerland are confronted with AS requests. Some institutions have started to develop their own guidelines on how to deal with AS within their walls, either refusing or allowing the performance of AS in their wards (Harding, 2005; Tuffs, 2007). In cases where suffering from mentalillness is the primary reason for requesting AS, the involvement and role of psychiatrists and other mental health care staff is likely. There is only one existing study exploring the support of physicians in Switzerland for AS under different medical scenarios, including dementia and refractory, chronic, severe mental illness (Brauer et al., 2015). The study was commissioned by the Swiss Academy of Medical Sciences (SAMS) to obtain an overview of the range of views held by physicians on the topic of AS and to inform new medical-ethical guidelines on 'Management of dying and death' (Swiss Academy of Medical Sciences (SAMS), 2018). In the survey, 28\% of all responding physicians supported the principle of AS, while $22 \%$ were clearly opposed to AS. $40 \%$ of the responding 
physicians believed that physicians should be allowed to perform AS (Brauer et al., 2015). Although the study shed some light on the attitudes of physicians practising in Switzerland, the low response rate of $34.9 \%$ and the wide variation of respondents from different professional specialties limited the generalizability of the survey's findings. In addition, the SAMS has recently revised its guidelines on physician-assisted suicide. The new guidelines propose the circumstances in which physician-assisted suicide can be ethically justifiable, most notably refraining from including the terminal illness criterion in the preconditions for AS (see their guidelines on 'Management of Death and Dying' (Swiss Academy of Medical Sciences (SAMS), 2018). This study aims at exploring psychiatrists' general support of the concept of AS in the context of SPMI, and regarding three different clinical scenarios presented in case vignettes.

[Insert Table 1 "The Swiss model” here]

\section{Research Questions}

To gather insight from psychiatrists practising in Switzerland, physicians with specialty training in psychiatry were asked about their attitudes towards AS requests by patients with SPMI, and specifically, their willingness to support patients in their decision to request AS. The first two research questions were therefore (1) if German-speaking psychiatrists in Switzerland support the possibility of AS in patients with SPMI in general and (2) whether they would take an active role in AS. Additionally, the psychiatrists were asked to evaluate three case vignettes of patients diagnosed with a severe and persistent mental illness: a patient with severe and enduring anorexia (vignette 1); a patient diagnosed with treatment-resistant depression (vignette 2); and a patient suffering from severe persistent schizophrenia (vignette 
3). The third research question was therefore (3) if the support is different for specific patients presented in case vignettes.

\section{Methods}

The quantitative cross-sectional survey was conducted in cooperation with the Swiss Society for Psychiatry and Psychotherapy (SSPP), and the protocol for it has been previously reported (Trachsel et al., n.d.). This study was outside the scope of the Swiss Human Research Act (HRA, Swiss Federal Council 2014) because no personal data concerning human diseases or concerning the structure or function of the human body were collected. However, the study has been assessed according to the Checklist for the ethical evaluation of empirical studies that do not need mandatory authorization (CEBES); No, CEBES-2016-04. Identities of subjects were completely anonymous ('blind survey') and the study was of a noninterventional nature. All participants received a copy of the survey by mail with an enclosed prepaid return envelope, and a reminder postcard to fill out the survey four weeks later.

\section{Sample}

The sample comprised all German-speaking members of the Swiss Society of Psychiatry and Psychotherapy (SSPP), corresponding to approximately 30 percent of psychiatrists in Switzerland. The sample size was $n=1311$. Data were collected in the period FebruaryMarch 2016.

\section{Survey and Case Vignettes}

This article reports five out of a total number of 42 items targeting other, different aspects in the care for severe and persistent mentally ill patients, in particular the suitability of palliative care approaches for this group of patients (Trachsel et al., n.d.). See Table 3 for all survey items. The two subsets of items are purposefully reported separately, since they are concerned 
with two distinct topics (palliative care approaches and assisted suicide in the context of SPMI). Participants were asked to respond to the statements on a 7-point Likert scale, ranging from completely disagree $(-3)$ to completely agree $(+3)$, with a neutral mid-point $(0)$. The case vignettes were previously published in different contexts (Trachsel, Wild, BillerAndorno, \& Krones, 2015; Brenner et al., 1990; Baweja \& Singareddy, 2013) and adapted to suit the goal and format of the survey. See Table 2 for the case vignettes. The participants had the opportunity to leave general comments in an optional 'General Comments' section. Participant comments were translated into English.

[Insert Table 2 "Case Vignettes” here]

[Insert Table 3 "Survey items" here]

\section{Statistical analysis}

Arithmetic means were calculated for age and work experience, and descriptive statistics (percentages) were calculated for gender, as well as for the Likert scale items. Analysis was undertaken using IBM SPSS Statistics 24.

\section{Patient and Public Involvement.}

This study aimed at investigating the attitude of psychiatrists, and there was no direct patient involvement in the development of the survey. However, the development was based on studies involving patients (e.g. the case vignettes). An advisory board consisting of experts from psychiatry, psychology, and palliative care was involved in all steps of this study. Further research on this topic involving the patient perspective is planned.

\section{Results}

\section{Sample characteristics}


The response rate was $34.9 \%$ ( 457 replies out of 1311 recipients of the survey). Mean age of the respondents was 57.8 years. $37 \%$ were female and $4.2 \%$ did not indicate their gender. The gender distribution reflected the total sample of active SSPP members $(62.9 \%$ male vs. $37.1 \%$ female). Mean work experience was 27.7 years (missing $n=23$ ).

\section{Attitudes towards assisted suicide on the basis of severe and persistent mental illness}

[Insert Figure 1 and 2 here]

Figure 1 and 2. Psychiatrists' attitudes on access and support of assisted suicide on the basis of a mental disorder.

$48.6 \%$ of the respondents stated that they do not generally support access to assisted suicide for persons diagnosed with an SPMI (rating $-1,-2$ or -3 on the scale, where -3 designated 'strongly disagree'). $21.2 \%$ of respondents remained neutral (rating ' 0 ' on the scale), while $29.3 \%$ stated that they do support for access to some degree (rating 1, 2 or 3 on the scale, where 3 designated 'strongly agree'). Missing $n=4(0.9 \%)$. There were no differences in age and work experience between respondents who remained neutral (rating ' 0 ' on the scale) and respondents who did not support/did support access to assisted suicide.

$55.6 \%$ of the respondents would not support a patient with SPMI in their AS request by writing a psychiatric report or referring them to a RTD organisation (rating $-1,-2,-3$ on the scale, where -3 designated 'strongly disagree'). $16.0 \%$ remained neutral (rating ' 0 ' on the scale) and $27.4 \%$ would provide support (rating 1, 2 or 3 on the scale, where 3 designated 'strongly agree'). Missing $n=5(1.1 \%)$.

\section{Responses to Vignettes}

Figure 3. Case vignettes. 
[Insert Figure 3 here]

$45.8 \%$ of respondents would not support the patient described in case vignette 1 in her explicit and enduring wish for assisted suicide by referring her to a respective organization (rating -1, -2 or -3 , where -3 designated strongly disagree). $17.5 \%$ remained neutral (rating ' 0 ' on the scale) and 35.4\% would provide support (rating 1, 2 or 3, where 3 designated strongly agree). Missing $n=6(1.3 \%)$.

$50.3 \%$ of respondents would not support the patient described in case vignette 2 in his explicit and enduring wish for assisted suicide by referring him to a respective organization. $16.6 \%$ remained neutral and $31.3 \%$ would provide support. Missing $n=8(1.8 \%)$.

$48.6 \%$ of respondents would not support the patient described in case vignette 3 in his explicit and enduring wish for assisted suicide by referring him to a respective organization. $17.7 \%$ remained neutral and 32.1\% would provide support. Missing $n=7(1.5 \%)$.

\section{Comments section}

Anecdotal quotes in the 'General Comments' section of the survey indicate that psychiatrists confronted with AS requests of their patients experience conflicts of interest. One respondent wrote: "As a psychiatrist, I would help to abolish my own profession if I would support

suicide." Another respondent stated: "I would only dare to adequately assess, if I had known the patient over a longer period of time, not on the basis of a single cross-sectional consultation. Generally, I do not support suicide in my position as a treating physician, irrelevant of my personal stance. Reason: I believe that my own personal judgement is dangerous because the patient initially had consulted me to improve his life. The patient, however, has the possibility to be aided in committing suicide, independently from me." 


\section{Discussion}

To our knowledge, this is the first study systematically assessing the attitudes of psychiatrists working in the medical landscape of Switzerland regarding the practice of AS in psychiatric patients. In light of the recent developments surrounding AS and psychiatry, this quantitative study is an important contribution to the current discourse.

\section{General support for AS in patients with SPMI}

While almost half of the respondents oppose the access to AS for patients with SPMI, more than a fifth of the respondents remains neutral ('0'), and $29.3 \%$ stated that they agree that patients with SPMI should have access to AS. This corresponds with a study among Swiss physicians of all subspecialties, where about $32 \%$ of respondents found AS to be justifiable for SPMI (Brauer et al., 2015). That same study revealed that psychiatrists do not have a higher or lower level of acceptance of AS for psychiatric patients than the general sample of medical providers (Brauer et al., 2015). A survey among Canadian psychiatrists' attitudes towards AS in the context of a primary psychiatric diagnosis found a similar number of psychiatrists $(29.4 \%)$ to be supportive, while the general support of AS in some circumstances (e.g. terminal illness) is $72 \%$ (Rousseau et al., 2017). Considering the current scholarly debate and the manifold objections raised by many experienced clinicians, the support in this survey for AS in patients with SPMI is considerable. Additionally, the percentage of those not choosing a clear stance (rating ' 0 ' on the scale) was high (between $16 \%$ and $21.2 \%$ for the different items). Importantly, there were no differences in age and gender for the "neutral" psychiatrists and the supporters/non supporters Although the interpretation of a neutral rating is speculative, this finding might be an indication of the conflicting roles and the ambivalence psychiatrists are currently experiencing with regard to AS (Miller \& Appelbaum, 2018). This ambivalence was stressed by some respondents in the 
comments' section. The practice of AS is often thought to be in conflict with the high priority of suicide prevention in psychiatry (Hewitt, 2013; Schuklenk \& van de Vathorst, 2015), as has been stressed by several authors in the comments' section. This is curious, since AS and classical suicide have traditionally been considered as two distinct phenomena. Only recently and solely within psychiatry scholars have started discussing a potential overlap. Specifically, it has been argued that psychiatric AS is difficult to distinguish from suicide due to shared characteristics and might ultimately affect the societal commitment to the prevention of suicide (S. Y. H. Kim, Conwell, \& Caine, 2018).

\section{Willingness to provide active support for psychiatric patients seeking AS}

$27.4 \%$ of the responding psychiatrists would actively support SPMI patients with concrete AS requests, e.g. by writing a psychiatric evaluation report or referring them to an RTDO. For the specific case vignettes, the willingness to provide support was even higher than on a general level (35.4\% for the patient with anorexia nervosa; $31.4 \%$ for the patient with schizophrenia; and $32.1 \%$ for the patient with depression). This is in contrast with a qualitative study among palliative care physicians in Switzerland, where establishing contact with the RTDOs was clearly perceived as the patients' responsibility (Gamondi et al., 2017).

\section{Lack of variation within diagnoses}

Studies show that the source of a patient's suffering can be decisive for the physician's willingness to grant AS requests, and that for a majority of physicians, physical symptoms are a precondition for 'unbearable' suffering (Bolt et al., 2015; Tol, Rietjens, \& van der Heide, 2010; Rietjens, Tol, Schermer, \& Heide, 2009). To our knowledge, there have been no studies regarding the variation of physician support for different psychiatric diagnoses. In this study, there was only small variation between the degree of support for the three different patients presented in the case vignettes. This is somewhat surprising, since the prognosis for the three 
presented patients varies, especially when it comes to fatal outcomes. While all three patients have an increased risk of death (Ivbijaro, 2017; Laursen, Nordentoft, \& Mortensen, 2014), patients with anorexia nervosa have the highest mortality rate of all mental illnesses (Arcelus J, Mitchell AJ, Wales J, \& Nielsen S, 2011). Additionally, the patient suffering from major depressive disorder currently has concrete suicidal intent, making a potential fatal outcome more likely. Interestingly, the presence of concrete suicidal intent did not significantly change the participating psychiatrists' willingness to support the depressed patient in his wish to seek AS (32.1\% support access to some degree). The lack of variation in diagnoses may be connected to the underlying general attitudes towards AS in psychiatric patients: participating psychiatrists either generally approved or disapproved of the practice as a whole.

\section{Strengths and Limitations}

The study has several strengths. The present study is the first to specifically assess the largely unexplored attitudes of psychiatrists towards AS in psychiatric patients in Switzerland through quantitative methods. By including different case vignettes, a more differentiated picture of their support for AS in patients with SPMI can be drawn.

The study also has several limitations. The nonresponse rate raises the possibility of a nonresponse bias. Age and gender, however, correspond to the total sample of SSPP members. Furthermore, we have not assessed other possible confounding variables such as field of work (e.g. private practice or hospital, adult versus geriatric psychiatry) or personal experience with AS. In addition, there is a risk of a sampling bias as only about $50 \%$ of all psychiatrists are members of the SSPP, and only German-speaking psychiatrists have been questioned in this survey. It is known that attitudes towards end-of-life practices differ between the language regions in Switzerland (Fischer et al., 2006). Yet, our results correspond with the findings from a recent questionnaire among Swiss physicians of all three language regions (Italian, French, and German), where $33 \%$ of all respondents tended to support AS in the case of 
patients with SPMI (Brauer et al., 2015). Finally, it is important to stress that Likert scale items allow no conclusions towards underlying concepts that influenced the psychiatrists' response behavior. We have chosen Likert scale items with a mid-point, rendering interpretation of a "neutral" midpoint difficult. On one hand, it is possible that respondents were undecided and ambivalent regarding their answers. On the other hand, respondents might have not concerned themselves enough, and therefore chose the mid-point. In this survey, the option to remain "neutral" was deliberately offered: We believe it is important to not force respondents to take side, avoiding possible frustration. This seems particularly important in the context of the controversial subject. Furthermore, it seems unlikely that respondents did not concern themselves enough to have a clear position since AS is a matter of increasing importance and high actuality in Switzerland.

\section{Implications for clinical practice and future directions}

In the few countries where AS based on a primary psychiatric diagnosis is legal, the numbers of patients seeking AS are consistently increasing (Dierickx, Deliens, Cohen, \& Chambaere, 2017; S. Kim \& Lemmens, 2016). Considering the ongoing global liberalization of AS practices and the likelihood of involvement by psychiatrists, particularly if the suffering derives from a mental illness, it is important to understand and include psychiatrists' perspectives. More research systematically assessing the reasons for support or disapproval, particularly including qualitative research, is necessary. The generated knowledge should inform formal education of psychiatrists on how to respond to requests and end-of-life issues in general, particularly where AS is a legal option for psychiatric patients.

In Switzerland, there is a particular urgency for developing a systematic approach to AS within mental health care in order to create adequate safeguard for the vulnerable group of patients with SPMI. The authors believe that psychiatrists who openly endorse the option of AS for some SPMI patients have an obligation to remain critical and take professional 
responsibility. Their involvement in the evaluation process (e.g. assessing decision-making capacity and assessing possible alternative options) is essential in order to ensure adequate safeguarding for SPMI patients. Those psychiatrists who generally refuse access of AS for SPMI should remain committed to the care for their AS-contemplating patients. Abandoning patients based on diverging moral beliefs is unethical and ultimately harmful to chronically suicidal patients. Furthermore, refusing to openly discuss the option of AS with SPMI patients in a value neutral environment is ultimately counterproductive in the care of suicidal SPMI patients. 


\section{References}

Appelbaum, P. S. (2016). Physician-assisted death for patients with mental disorders-reasons for concern. JAMA Psychiatry, 73(4), 325-326. https://doi.org/10.1001/jamapsychiatry.2015.2890

Arcelus J, Mitchell AJ, Wales J, \& Nielsen S. (2011). Mortality rates in patients with anorexia nervosa and other eating disorders: A meta-analysis of 36 studies. Archives of General Psychiatry, 68(7), 724-731. https://doi.org/10.1001/archgenpsychiatry.2011.74

Baweja, R., \& Singareddy, R. (2013). Concomitant use of maintenance ECT and vagus nerve stimulation for more than 10 years in treatment-resistant depression. American Journal of Psychiatry, 170(9), 1059-1061. https://doi.org/10.1176/appi.ajp.2013.13010125

Bolt, E. E., Snijdewind, M. C., Willems, D. L., Heide, A. van der, \& Onwuteaka-Philipsen, B. D. (2015). Can physicians conceive of performing euthanasia in case of psychiatric disease, dementia or being tired of living? Journal of Medical Ethics, 41(8), 592-598. https://doi.org/10.1136/medethics-2014-102150

Brauer, S., Bolliger, C., \& Strub, J.-D. (2015). Swiss physicians' attitudes to assisted suicide: A qualitative and quantitative empirical study, $145,1-8$. https://doi.org/10.4414/smw.2015.14142

Brenner, H. D., Dencker, S. J., Goldstein, M.J., Hubbard, J. W., Keegan, D. L., Kruger, G., ... Midha, K. K. (1990). Defining treatment refractoriness in schizophrenia. Schizophrenia Bulletin, 16(4), 551-561. https://doi.org/10.1093/schbul/16.4.551

Dierickx, S., Deliens, L., Cohen, J., \& Chambaere, K. (2017). Euthanasia for people with psychiatric disorders or dementia in Belgium: analysis of officially reported cases. BMC Psychiatry, 17. https://doi.org/10.1186/s12888-017-1369-0

Dyer, O., White, C., \& Rada, A. G. (2015). Assisted dying: law and practice around the world. $B M J$, 351, h4481. https://doi.org/10.1136/bmj.h4481

Emanuel, E. J., Onwuteaka-Philipsen, B. D., Urwin, J. W., \& Cohen, J. (2016). Attitudes and practices of euthanasia and physician-assisted suicide in the United States, Canada, and Europe. JAMA, 316(1), 79-90. https://doi.org/10.1001/jama.2016.8499 
Fischer, S., Bosshard, G., Faisst, K., Tschopp, A., Fischer, J., Bär, W., \& Gutzwiller, F. (2006). Swiss doctors' attitudes towards end-of-life decisions and their determinants: a comparison of three language regions. Swiss Medical Weekly, 136(23-24), 370-376. https://doi.org/2006/23/smw11260

Gamondi, C., Borasio, G. D., Oliver, P., Preston, N., \& Sheila Payne. (2017). Responses to assisted suicide requests: an interview study with Swiss palliative care physicians. BMJ Supportive \& Palliative Care, 0, 1-9. https://doi.org/10.1136/bmjspcare-2016-001291

Harding, L. (2005, December 19). Swiss hospital the first to allow assisted suicides. Retrieved February 12, 2018, from http://www.theguardian.com/society/2005/dec/19/health.medicineandhealth

Hewitt, J. (2013). Why are people with mental illness excluded from the rational suicide debate? International Journal of Law and Psychiatry, 36(5), 358-365. https://doi.org/10.1016/j.ijlp.2013.06.006

Ivbijaro, G. (2017). Excess mortality in severe mental disorder: the need for an integrated approach. World Psychiatry, 16(1), 48-49. https://doi.org/10.1002/wps.20382

Kim, S., \& Lemmens, T. (2016). Should assisted dying for psychiatric disorders be legalized in Canada? Canadian Medical Association Journal, 188(14), 337-339. https://doi.org/10.1503/cmaj.160365

Kim, S. Y. H., Conwell, Y., \& Caine, E. D. (2018). Suicide and physician-assisted death for persons with psychiatric disorders: How much overlap? JAMA Psychiatry, 75(11), 1099-1100. https://doi.org/10.1001/jamapsychiatry.2018.2065

Kouwenhoven, P. S., Raijmakers, N. J., van Delden, J. J., Rietjens, J. A., Schermer, M. H., van Thiel, G. J., ... van der Heide, A. (2013). Opinions of health care professionals and the public after eight years of euthanasia legislation in the Netherlands: A mixed methods approach. Palliative Medicine, 27(3), 273-280. https://doi.org/10.1177/0269216312448507

Laursen, T. M., Nordentoft, M., \& Mortensen, P. B. (2014). Excess early mortality in schizophrenia. Annual Review of Clinical Psychology, 10(1), 425-448. https://doi.org/10.1146/annurevclinpsy-032813-153657 
Miller, F. G., \& Appelbaum, P. S. (2018). Physician-assisted death for psychiatric patients misguided public policy. New England Journal of Medicine, 378(10), 883-885. https://doi.org/10.1056/NEJMp1709024

Regional euthanasia review committees. (2017). Annual reports. Retrieved January 15, 2019, from https://english.euthanasiecommissie.nl/the-committees/documents/publications/annualreports/2002/annual-reports/annual-reports

Rietjens, J. a. C., Tol, D. G. van, Schermer, M., \& Heide, A. van der. (2009). Judgement of suffering in the case of a euthanasia request in The Netherlands. Journal of Medical Ethics, 35(8), 502507. https://doi.org/10.1136/jme.2008.028779

Rousseau, S., Turner, S., Chochinov, H. M., Enns, M. W., \& Sareen, J. (2017). A national survey of Canadian psychiatrists' attitudes toward medical assistance in death. The Canadian Journal of Psychiatry, 62(11), 787-794. https://doi.org/10.1177/0706743717711174

Schuklenk, U., \& van de Vathorst, S. (2015). Treatment-resistant major depressive disorder and assisted dying. Journal of Medical Ethics, 41(8), 577-583. https://doi.org/10.1136/medethics$2014-102458$

Schuklenk, U., \& Vathorst, S. van de. (2015). Treatment-resistant major depressive disorder and assisted dying: response to comments. Journal of Medical Ethics, 41(8), 589-591. https://doi.org/10.1136/medethics-2015-102966

Steck, N., Junker, C., \& Zwahlen, M. (2018). Increase in assisted suicide in Switzerland: did the socioeconomic predictors change? Results from the Swiss National Cohort. BMJ Open, 8(4), e020992. https://doi.org/10.1136/bmjopen-2017-020992

Swiss Academy of Medical Sciences (SAMS). (2018, May). Management of dying and death. Retrieved from https://www.samw.ch/en/Ethics/Ethics-in-end-of-life-care/Guidelinesmanagement-dying-death.html

Tol, D. van, Rietjens, J., \& van der Heide, A. (2010). Judgment of unbearable suffering and willingness to grant a euthanasia request by Dutch general practitioners. Health Policy, 97(2), 166-172. https://doi.org/10.1016/j.healthpol.2010.04.007 
Trachsel, M., Hodel, M. A., Irwin, S. A., Hoff, P., Biller-Andorno, N., \& Riese, F. (n.d.).

Acceptability of palliative care approaches for patients with severe and persistent mental illness: A survey of psychiatrists in Switzerland. Submitted.

Trachsel, M., Wild, V., Biller-Andorno, N., \& Krones, T. (2015). Compulsory treatment in chronic anorexia nervosa by all means? Searching for a middle ground between a curative and a palliative approach. The American Journal of Bioethics, 15(7), 55-56. https://doi.org/10.1080/15265161.2015.1039730

Tuffs, A. (2007). Swiss hospitals admit to allowing assisted suicide on their wards. British Medical Journal, 335(7629), zie. https://doi.org/10.1136/bmj.39405.470498.94

Vandenberghe, J. (2018). Physician-assisted suicide and psychiatric illness. New England Journal of Medicine, 378(10), 885-887. https://doi.org/10.1056/NEJMp1714496

Ziegler, S. J. (2009). Collaborated death: An exploration of the Swiss model of assisted suicide for its potential to enhance oversight and demedicalize the dying process. The Journal of Law, Medicine \& Ethics, 37(2), 318-330. https://doi.org/10.1111/j.1748-720X.2009.00375.x

Ziegler, S. J., \& Bosshard, G. (2007). Role of non-governmental organisations in physician assisted suicide. British Medical Journal, 334(7588), 295-298. https://doi.org/10.1136/bmj.39100.417072.BE

\section{Funding statement}

This work was supported by the Palliative Care Research funding program of the Swiss Academy of Medical Sciences (SAMS); the Gottfried and Julia Bangerter-Rhyner Foundation; and the Stanley Thomas Johnson Foundation. FR received funding through Swiss National Science Foundation grant 406740_139363. The views expressed in this paper are those of the authors and do not necessarily reflect those of the funder. The funding body in no way influenced the authors in writing the manuscript.

\section{Competing interests of all authors}


None.

\section{Authors' contribution/acknowledgements}

MAH and MT constructed and conducted the survey; MAH analysed the data and drafted the manuscript. All authors were involved in the study's conception and design, interpretation of the data, critical revision of the article and final approval of the version submitted for publication.

We would like to thank the Swiss Society of Psychiatry and Psychotherapy, and especially vice-president Daniel Bielinski (MD), for their support in the acquisition of data. We also thank Roland Kunz (MD) for his conceptual input. 